\title{
Research Paper \\ The Effect of an 8-Week Yoga Training Program on Balance and Knee Proprio- ception After Mental Fatigue in Amateur Male Athletes
}

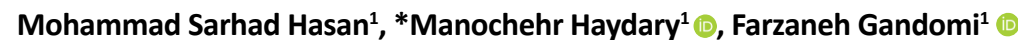

1. Department of Corrective Exercises and Sport Injuries, Faculty of Physical Education, Razi University, Kermanshah, Iran.

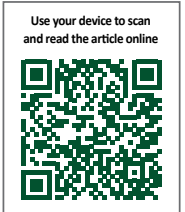

cttation: Sarhad Hasan M, Haydary M, Gandomi F. [The Effect of an 8-Week Yoga Training Program on Balance and Knee Proprioception After Mental Fatigue in Amateur Male Athletes (Persian)]. Journal of Sport Biomechanics. 2020; 5(4):228-239.

doi'https://doi.org/10.32598/biomechanics.5.4.3

Key words:

Yoga, Mental fatigue, Balance, Proprioception

\section{ABSTRACT}

\section{Article Info:}

Received: 19 Jan 2019

Accepted: 13 Oct 2019

Available Online: 01 Mar 2020
Objective Mental fatigue is caused by long-term cognitive activities and lead to changes in motor coordination. The purpose of this study was to investigate the effect of yoga training on balance and knee proprioception after mental fatigue in amateur male athletes.

Methods In this quasi-experimental study, 36 amateur male students were voluntarily selected as study samples and randomly divided into training $(n=18)$ and control $(n=18)$ groups. The training group received yoga exercises for eight weeks, three sessions per week, each for one hour. Mental fatigue was induced by 45 minutes of cognitive activity (Stroop test). Before and after mental fatigue and intervention, their dynamic balance was measured by Y-balance test and the semi-dynamic balance by Lafayette stability platform. Data were analyzed in SPSS v.22 software using paired sample t-test at the significant level of 0.05 . Results The effects of time and groupxtime interaction on dynamic and semi-dynamic balances $(P=0.0001)$, and on active and passive knee proprioception $(P=0.0001)$ were significant after yoga training. Conclusion There were significant improvements in balance and knee proprioception of amateur athletes after yoga training. Further studies should be conducted to introduce solutions to the occurrence of mental fatigue in these athletes.

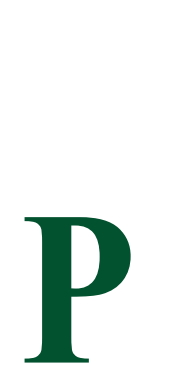

Extended Abstract

\section{Introduction}

roper lower limb function is one of the most essential factors of participating in sports activities $[1,2]$. Most of the ligament injuries have occurred during landings and rotations, as the most important mechanisms in the occurrence of lower limb injuries [3]. Functional stability is one of the essential indicators for participating in sports [3], and plays an important role in people's activities, including simple daily tasks and highlevel performances in sports based on their nature [4]. In this regard, proprioception as a very effective part of somatosensory system, plays a significant role in maintaining the balance of people. Therefore, any weakness and impairment in proprioception significantly increases the risk of injuries to athletes [5].

Since proprioception sense is responsible for collecting information for the central nervous system to be informed of different parts of the body in relation to each other, any factor

\section{* Corresponding Author:}

Manochehr Haydary, MSc.

Address: Department of Corrective Exercises and Sport Injuries, Faculty of Physical Education, Razi University, Kermanshah, Iran.

Tel: +98 (912) 5382491

E-mail: mhaidary2000@yahoo.com 
that disrupts this sense is a cause of damage and must be controlled. One of the factors that impair the proprioception sense is fatigue, because it increases the threshold of muscle spindle discharge, disrupting afferent feedback, and causing change in the joint awareness [6]. Studies have shown that in the onset of fatigue, neuromuscular control is impaired and due to delayed neuromuscular activation, shear forces and torque increase which impair joint stability [7, 8]. Many sports that require cognitive activity are prone to mental fatigue; however, lower limb injuries in these sports are high. Due to the considerable effect of mental fatigue on the occurrence of injury and the fact that no research has been done in this field so far, this study aimed to evaluate the effect of yoga training on knee proprioception and balance after mental fatigue in amateur athletes.

\section{Methods}

In this quasi-experimental study with pretest/posttest design using control group, the study population consisted of all male physical education students of Razi University in Kermanshah, Iran during the second semester. Of these, 36 were selected as study samples and were divided into two groups of training $(\mathrm{n}=18)$ and control $(\mathrm{n}=18)$ randomly using Random Number Allocation software. The training group performed yoga for 8 weeks, while control group received no intervention. Study outcomes included: dynamic balance (Y-test), semi-dynamic balance (Lafayette Stability Platform) and knee joint proprioception (reconstruction error at 30 and 60 degrees angles). Each subject was assessed in three stages: before the intervention, after the fatigue protocol, and eight weeks after receiving the mental fatigue protocol (follow-up). To compare the mean values, repeated measures ANOVA (two groups in three times) was used. All statistical analyzes were performed in SPSS v. 22 software.

\section{Results}

For active and passive knee proprioception as well as static and dynamic balances, repeated measures ANOVA results showed that the effect of time $(\mathrm{P}=0.001)$ and time-group interaction $(\mathrm{P}=0.001)$ was significant. Moreover, the results of Benfroni post hoc test showed that these changes between pretest and posttest phases and between posttest and followup phases were statistically significant $(\mathrm{P}=0.001)$.

\section{Discussion}

The results of this study showed that yoga training inhibit mental fatigue by increasing the adaptive capacity of neurocognitive systems and modulating the autonomic nervous system, and can improve the balance function and knee joint proprioception by creating physical stability. It is possible that yoga helps to increase adaptation by reducing stress, and brings peace of body and mind to humans by limiting tension and reducing arousal in the cerebral cortex [9]. Some sports scientists have considered the fatigue issue as one of the negative factors affecting athletic performance and its relationship with postural control, because fatigue and impaired postural control can be causes of musculoskeletal injuries in athletes [10].

Martino et al. reported that after mental fatigue, due to the accumulation of adenosine in the brain, increased resistance to effort, feeling and perception of fatigue and lack of energy were observed [11]. Some other researchers have reported a quantitative and qualitative decrease in the technical performance of footballers due to mental and physical fatigue. Kastem et al. reported that endurance performance (time to reach fatigue, ability to speed up activity) decreased as a result of mental fatigue [12]. The results of these studies are consistent with our findings.

\section{Conclusion}

Yoga exercise can significantly improve balance and knee joint proprioception in amateur athletes. Mental fatigue is caused by long-term cognitive activities; it can affect balance control and perception of joint position and movement, and reducing the performance of athletes by exposing them to lower limb injuries. Therefore, doing yoga exercises to control and raise the threshold of mental fatigue can be a suitable intervention.

\section{Ethical Considerations}

\section{Compliance with ethical guidelines}

All ethical principles are considered in this article. This study was approved by the Ethics Committee of Razi University (Code: IR.RAZI.REC.1398.001).

\section{Funding}

The present paper was extracted from the MSc. thesis of the first author, Department of Corrective Exercises and Sport Injuries, Faculty of Physical Education, Razi University.

\section{Authors' contributions}

All authors contributed in preparing this article.

\section{Conflicts of interest}

The authors declared no conflict of interest. 


\section{Acknowledgements}

Thus, authors thanks and appreciation the Razi University Physical Education Administration and the physical education students who participated in the test. 


\title{
اثر هشت هفته تمرين يوكًا بر كنترل خستكًى ذهنى و حس عمقى زانو و تعادل تغييريافته در

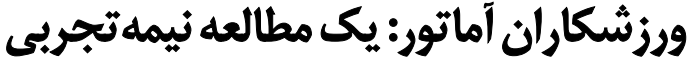

\author{
محمد سر حد حسن'! 'منوجهر حيدرى' (1) فرزانه كندمى \\ ا. كروه آسيب شناسى ورزشى و حركات اصلاحى، دانشكده علوم ورزشى، دانشُاه رازي، كرمائشاه، ايران.
}

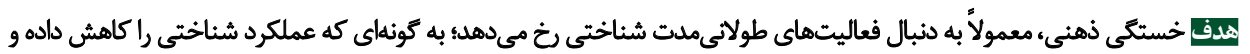

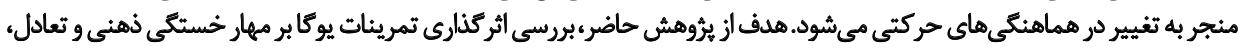

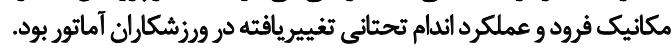

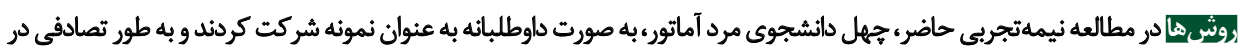

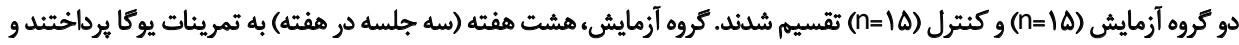

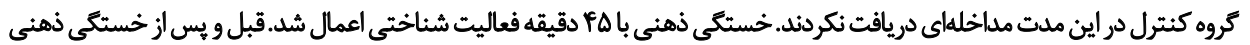

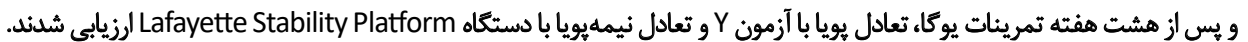

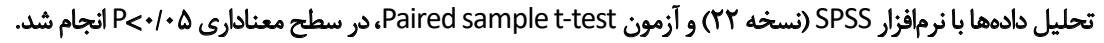

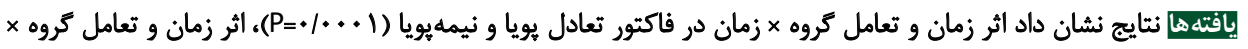

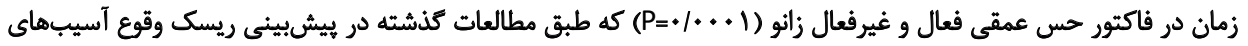

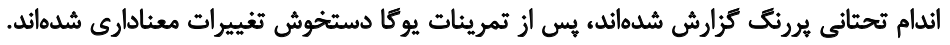

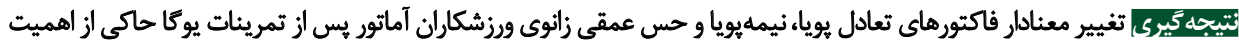

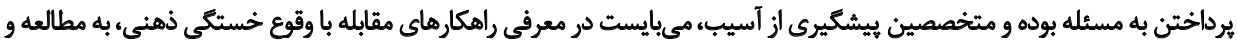

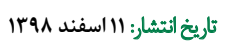

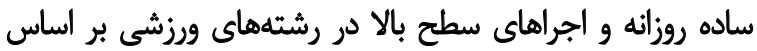

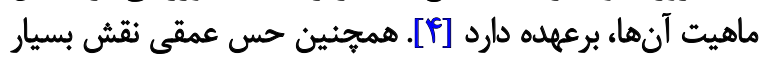
امروزه اكثر آسيبهاى ورزشى به دنبال برخورد فيزيكى مستقيم إيجاد زيادى در حفظ تعادل افرواد دارد.

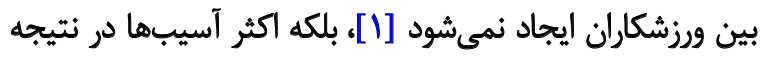

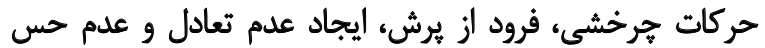

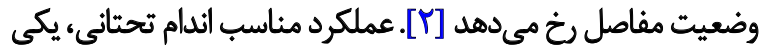
تأثير نسبى حس عمقى و سيستم وستيبولار 'ر ادرغياب سيستم

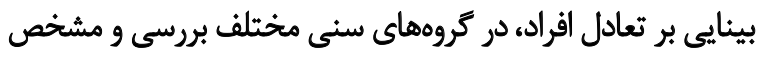

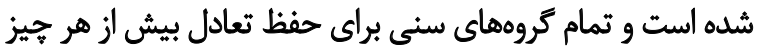

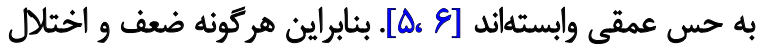

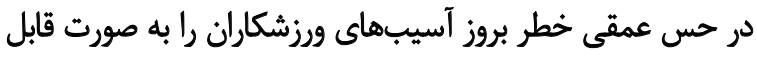

1. Proprioception

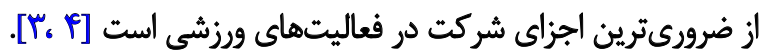

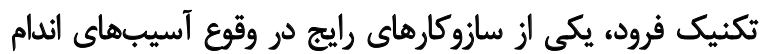

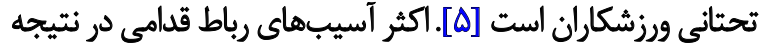

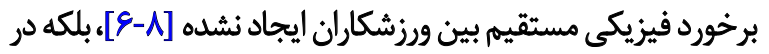

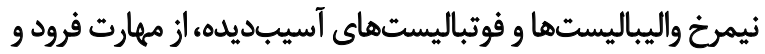

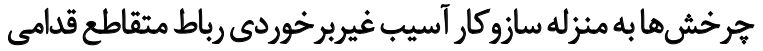

2. Vestibular system 
يافتههاي تحقيقات حاكى ازٔ آن است كه تمرينات يوكا باعث افزايش

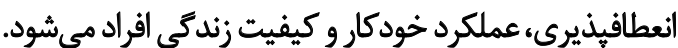
از سويى ديكر بسيارى از رشتهائ ورزشى مثل فوتبال، بسكتبال،

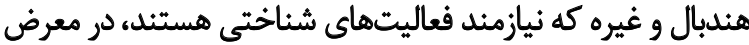

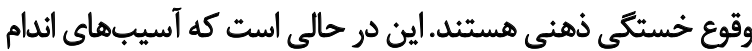

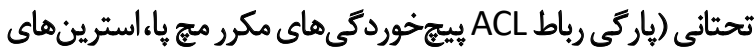

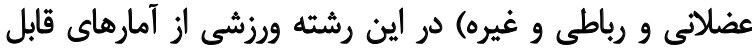
توجهى برخوردار است.

با توجه به اينكه از يك طرف دستورالعملهاي موجود براى

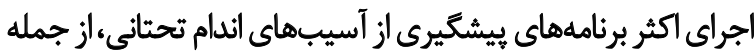

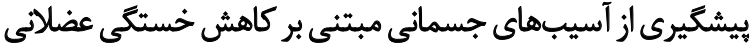

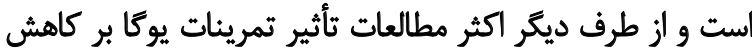

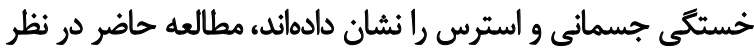

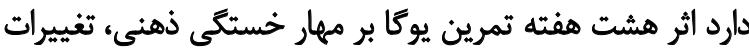

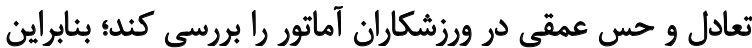

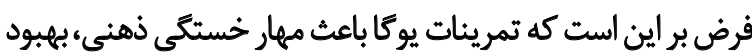
تغييرات، تعادل و كنترل حس عمقى مىشود.

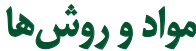

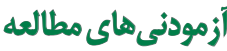

تحقيق حاضر، يك مطالعه نيمهتجربى يكسويهور با طرح

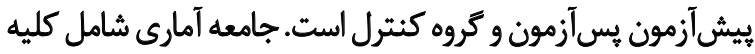

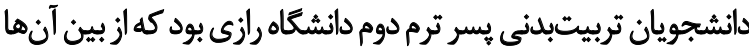

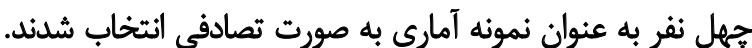

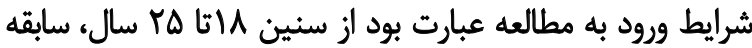

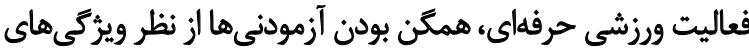

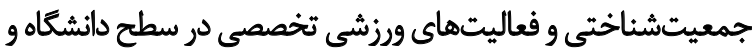

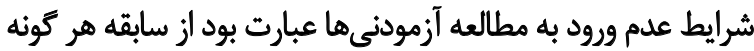

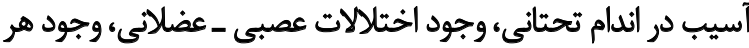

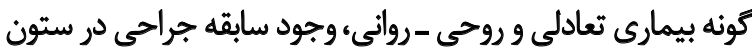

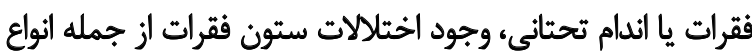

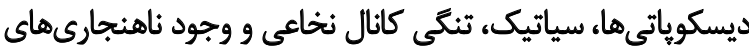

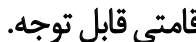

تعداد آزمودنى ها بر اساس نرم افزار

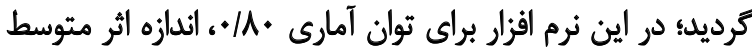

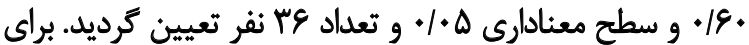

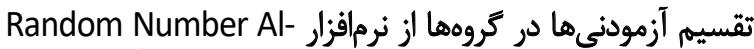

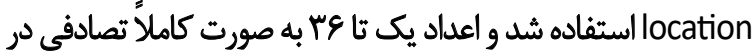

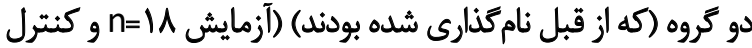
(n=11 در اين نرمافزار قرار كرفتند.

ليست اعداد در اختيار فردى غيردركير در كار تحقيقى قرار

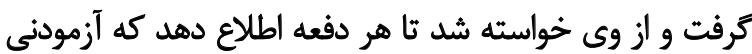

توجهي افزايش مي دهد [V]. از طرفى حس عمقى بخشى از سيستم

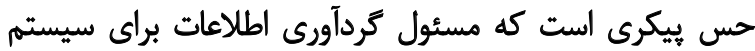

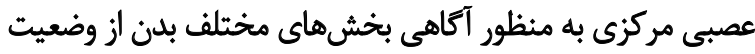

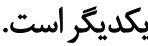

يكى از عوامل ايجاد اختلال در حس عمقى خستكى است؛ زيرا

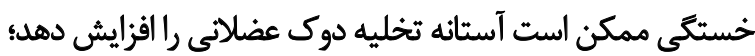

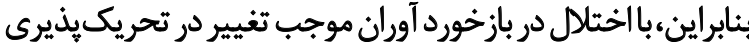

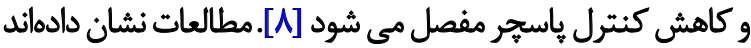

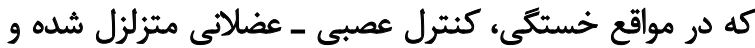

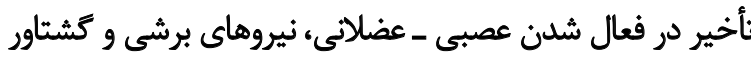

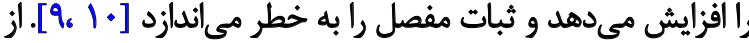

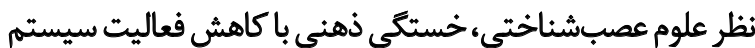

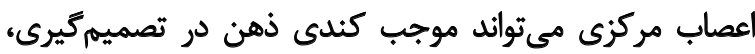

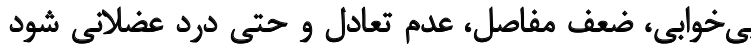

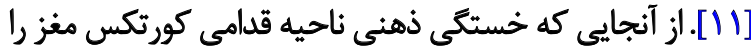

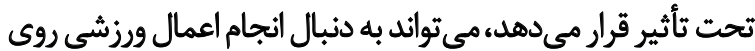

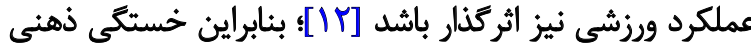

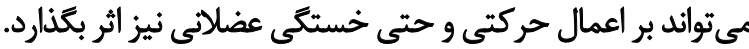

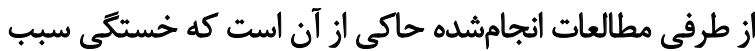

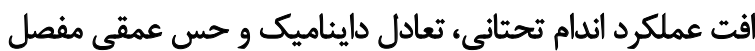

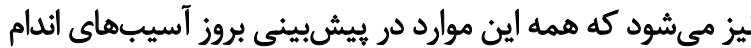
تحتانى نقش مؤثرى دارئد.

در همين راستا خسروى و همكاران و كَندمى و همكاران

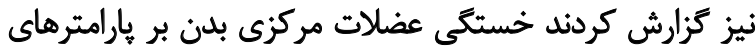

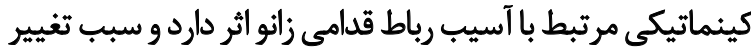

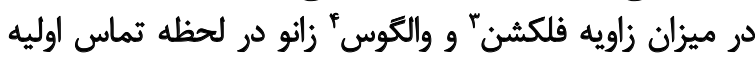

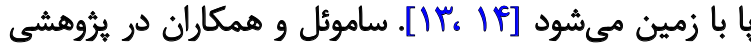

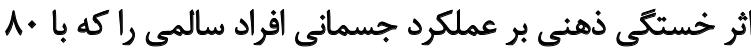

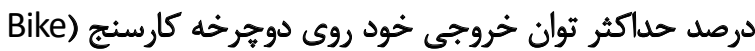

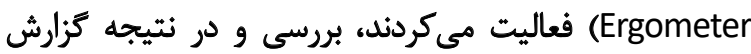

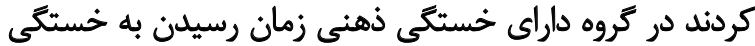

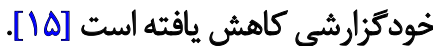
يوكا به عنوان يك نظريه شخصيت نيز در نظر ترفته مي شيود كه

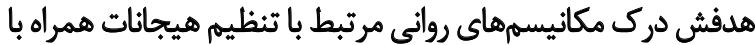

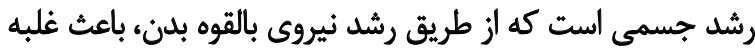

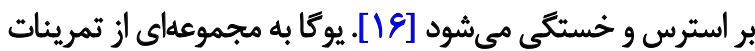

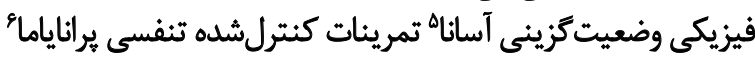

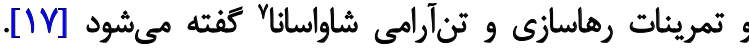

3. Flexion

4. Valgus

5. Asana

6. Pranayama

7. Shavasana 


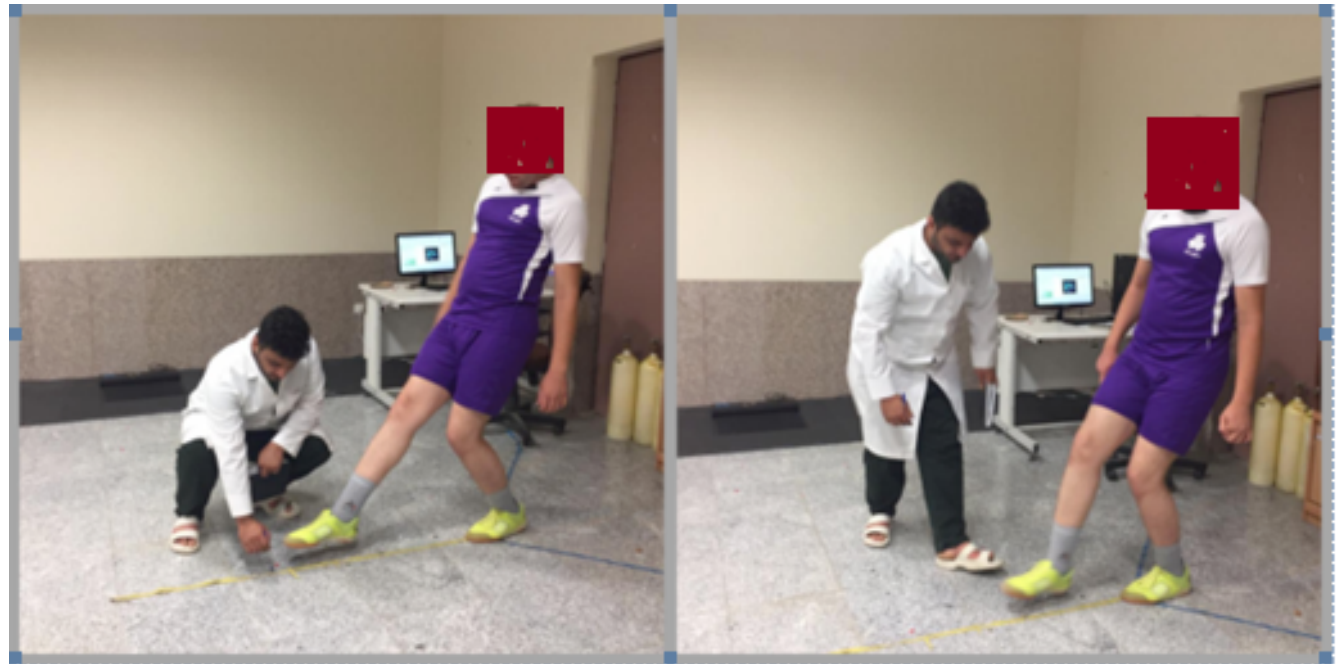

مجله بيومكانيك ورنث

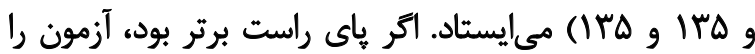

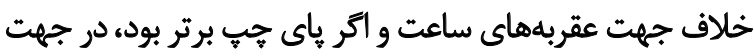

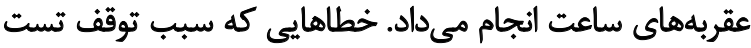

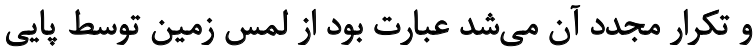

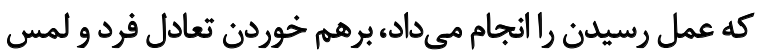

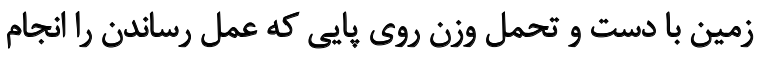

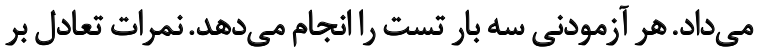

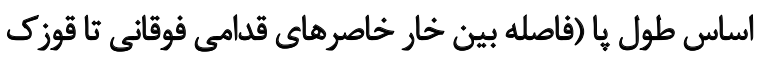

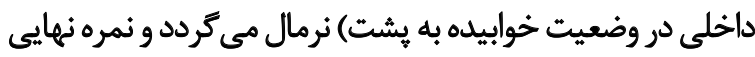

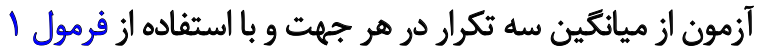

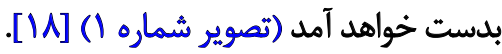

1. نمره تعادل يويا = مسافت طى شده از سه جهت / طول يا ×
تصوير I. نحوه ايدازمكيرى تست تعادل

ارزيابىشده در كدام كروه قرار مى كيرد. بدينترتيب ارزياب از

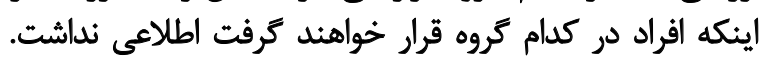

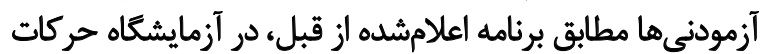

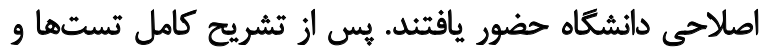

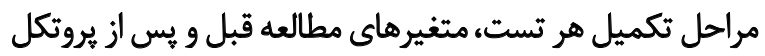

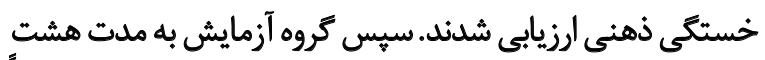

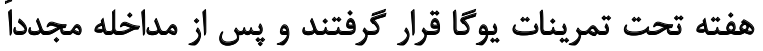

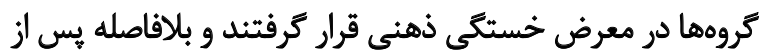
آن متغيرها براى بار سوم اندازهيرى شئى ذرند.

تعادل يوياى Y: يك آزمون معتبر براي ارزيابى تعادل يوياست

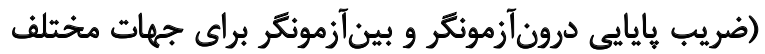

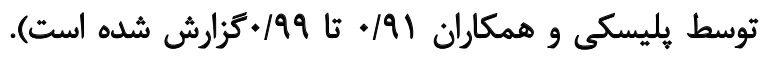

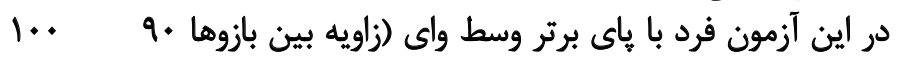

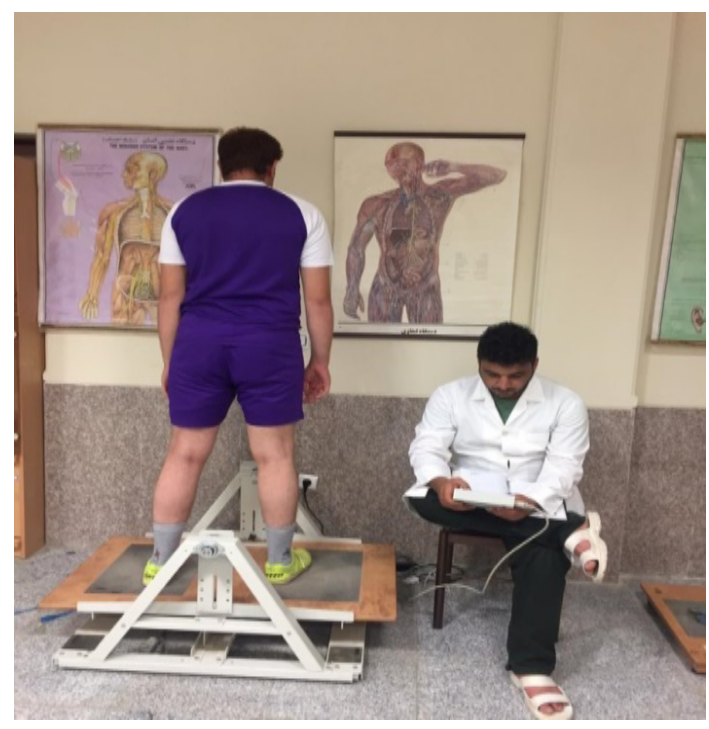


به منظور دقت بيشتر اندازهيرى، آزمون بازسازى زاويه سهبار

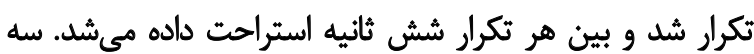

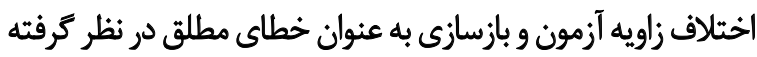

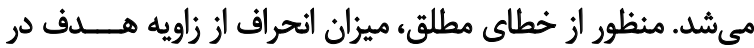

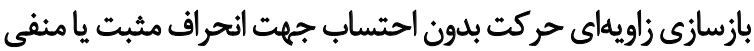

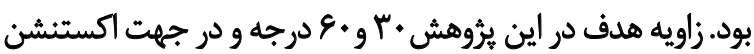

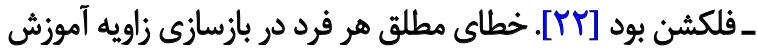
دادهشه، به عنوان متغير وابسته در نظر كرفته شد.

$$
\text { روشها و تجزيه و تحليل آمارى }
$$

جهت بررسى توصيف دادهها و رسم نمودارها از آمار توصيفى و

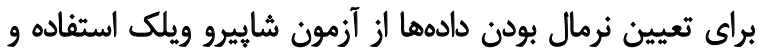

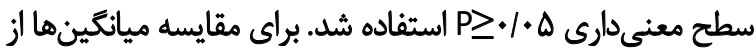

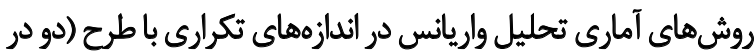

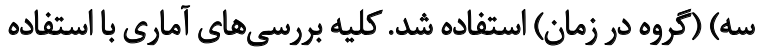

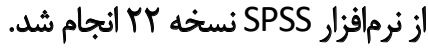

\section{نتايج}

اطلاعات توصيفى آزمودنىها شامل ميانكين و انحراف

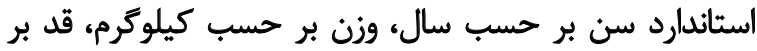

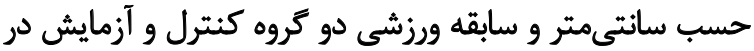
جدول شماره إرائه شده است.

$$
\text { حس عمقى فعال زانو }
$$

همان طور كه از جدول شماره ب برمى آيد، يافتههاى آزمون تحليل

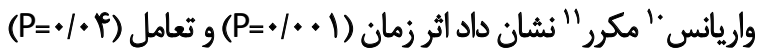

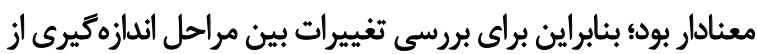

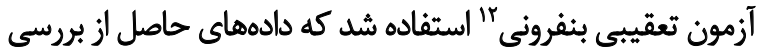

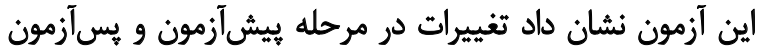

10. Variance

11. Repeated measure

12. Bonfroni
تعادل نيمهايويا توسط صفحه نايايدارث، آزمودنى ابتدا يك دقيقه

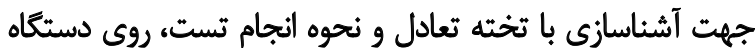

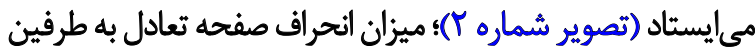

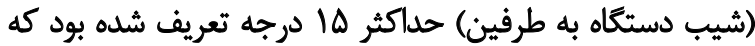

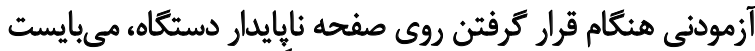

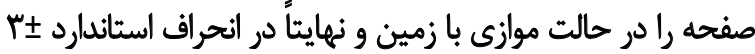

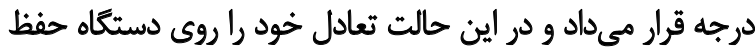

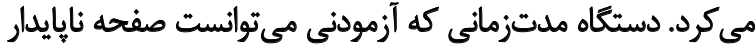

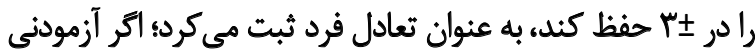

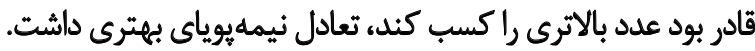

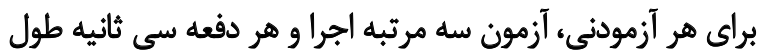

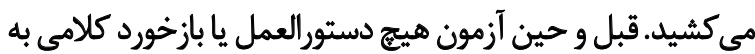

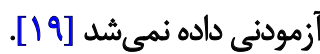

$$
\text { ازويابى حس عمقى مفصل زانو }
$$

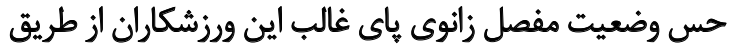

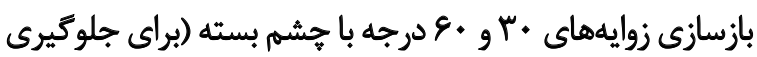

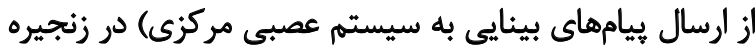

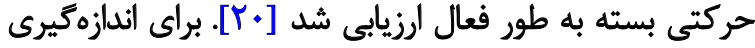

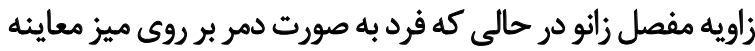

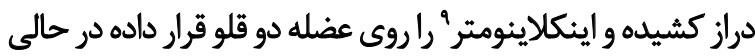

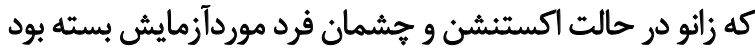

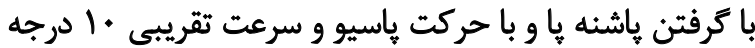

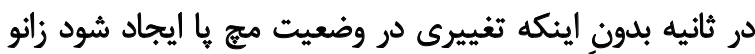

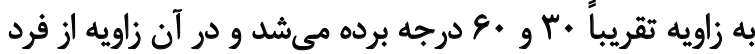

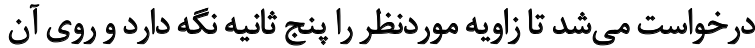

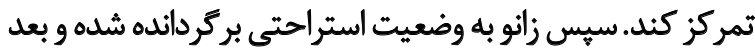

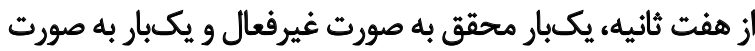

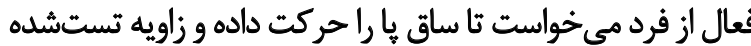

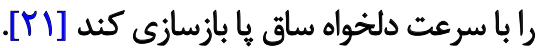

8. Lafayette Stability Platform with Digital Control

\begin{tabular}{|c|c|}
\hline ميانئين||نحراف معيار & ويرؤى \\
\hline$r \cdot / \Delta \pm r / /$ & سن (سال) \\
\hline$e 9 / V \pm 11 /$ & وزن (كيلوكرم) \\
\hline IVNTE\&/A & قد (سائتىمثر) \\
\hline$r / r \pm r / r$ & سايقه ورزشى (سال) \\
\hline
\end{tabular}

9. Inclinometer

جدول ا. ويزّكى هاى جمعيتشُناختى آزمودنى ها (N=هr)

مجله بيومكانيك ورنث 
جدول ب. آزمون تعقيبى بثفرومى جهت ارزيابى اثير تغييرات مراحل اندازميرى حس عمقى فعال زائو

\begin{tabular}{|c|c|c|c|}
\hline سطع معنادارى & اختلاف ميانئين & زمان & زمان \\
\hline $.1 ..)^{*}$ & $-r$ & يسآلزمون اول & ييش آزمون \\
\hline $.1 . .1^{*}$ & 1 & يس آزمون دوم & يسآزمون الول \\
\hline$\cdot 1 \cdot \cdot \Delta^{*}$ & -1 & يس آزهون دوم & ييش آزمهون \\
\hline
\end{tabular}

جدول r. آزمون تعقيبى بنفرومى جهت ارزيابى اثر تغييرات مراحل اندازهيرى حس عمقى غيرفعال زانو

\begin{tabular}{|c|c|c|c|}
\hline سطح معنادارى & اختلاف ميانكين & زمان & زمان \\
\hline $.1+e^{e *}$ & -1 & هسآزمون اول & يبش أزمون \\
\hline $.1 . .1^{*}$ & $-r$ & يسآزهون دوم & يسآزهون الول \\
\hline $.1 .01^{*}$ & -1 & يس آزمهن دوم & ييش آزمون \\
\hline
\end{tabular}

جدول P. آزمون تعقيبى بنفرومى جهت ارزيابى اثر تغييرات مراحل اندازهيرى بر تعادل يويا

\begin{tabular}{|c|c|c|c|}
\hline سطح محنادارى & اختثالف ميانكين & زمان & زمان \\
\hline.$/+*$ & 10 & يسآزمون اول & ييشأزمون \\
\hline$+1+.1^{*}$ & $-r$ & يس آزمون دوم & يسأزمون اول \\
\hline$+1 . .1^{*}$ & if & يسآزمون دوم & ييش أزمون \\
\hline
\end{tabular}

جدول هـ. آزمون تعقيبى بنفرومى جهت ارزيابى اثر تغييرات مراحل اندازهيرى بر تعادل نيمهيويا روى صفحه نإيايدار يلتفرم

\begin{tabular}{|c|c|c|c|}
\hline سطح معنادارى & اختالف ميانعين & مرحله & مرحله \\
\hline $.1 . .1^{*}$ & $f$ & يسآزمون اول & ييش أزمون \\
\hline.$+.1^{*}$ & - & يس آزمون دوم & يسأزمون اول \\
\hline$+1+\infty 1^{*}$ & i & هس آزمون دوم & ييش أزمون \\
\hline
\end{tabular}

كه خستكى ذهنى بر حس عمقى زانو اثركذار بود و با ايجاد خستكي

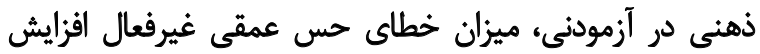

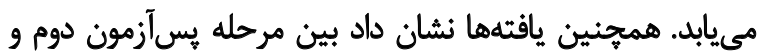

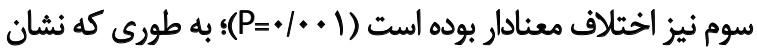

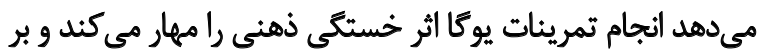
عملكرد حس عمقى غيرفعال زانو اثر مثبت دارد.

\section{Yتعادل يوياى}

يافتههاى آزمون تحليل واريانس مكرر نشان داد اثر زمان

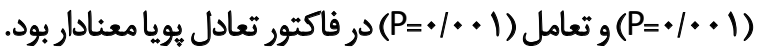

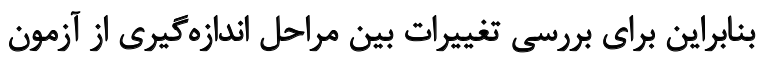

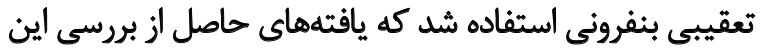

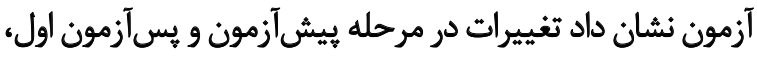

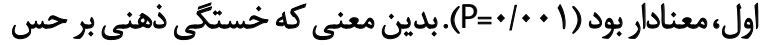

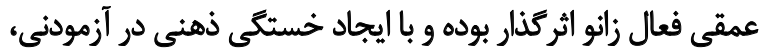

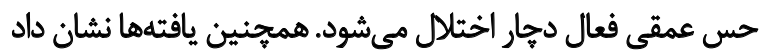

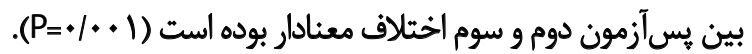

$$
\text { حس عمقى غيرفعال زانو }
$$

همان طور كه از جدول شماره ب برمى آيد، يافتههاي آزمون تحليل

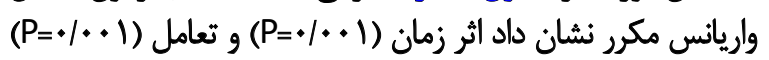

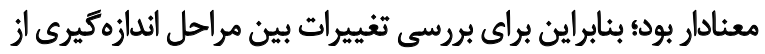

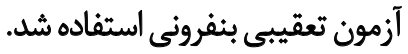
دادههاى حاصل از بررسي اين آزمون نشان داد تغييرات در مرحله

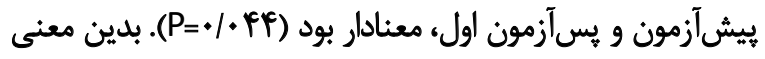


تأثير كذار باشد، به كونهاى كه ميزان خطاى بازسازى زاويه هدف در

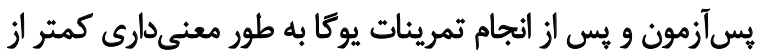

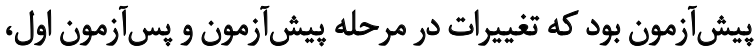

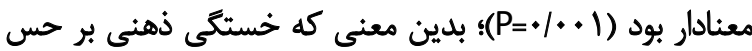

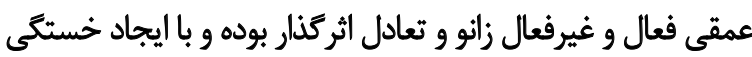

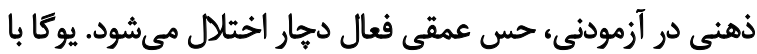

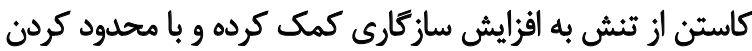

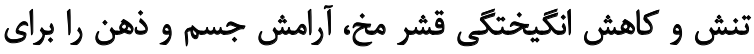

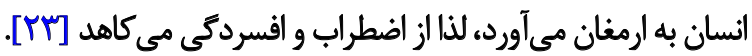

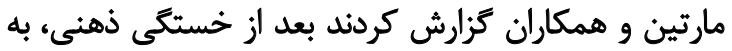

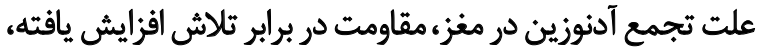

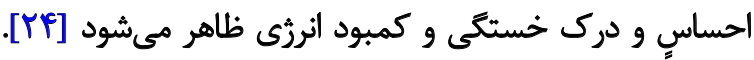

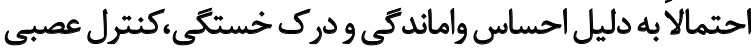

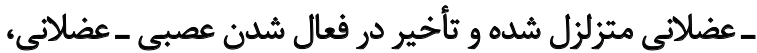

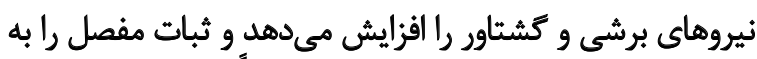

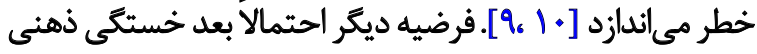

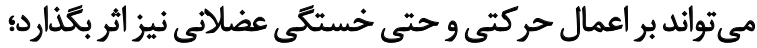

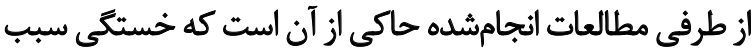

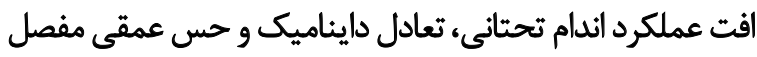

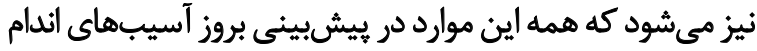
تحتائى نقش مؤثرى دارند.

در اين زمينه، تحقيقى كه اثر تمرينات يوكا بر مهار خستكى تماتى

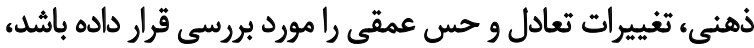

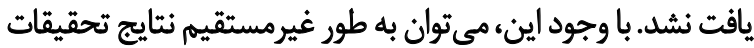

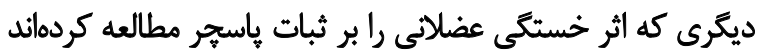

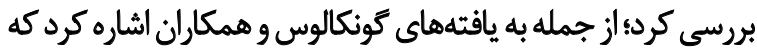

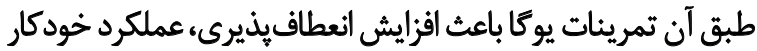

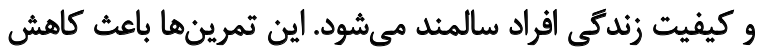

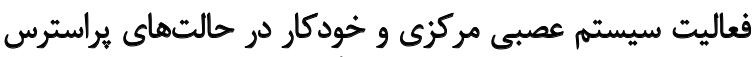

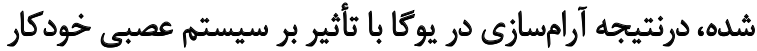

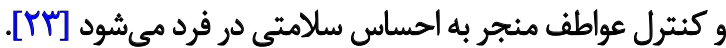
بارنى و همكاران نيز در تحقيق خود كه فوتباليستها را مورد

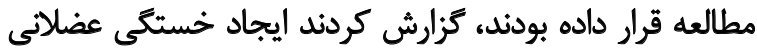

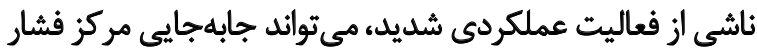

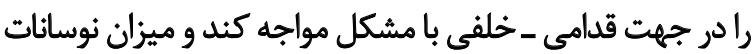

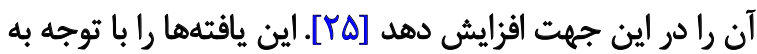

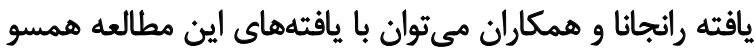

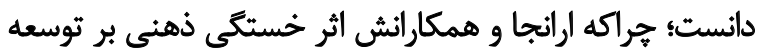

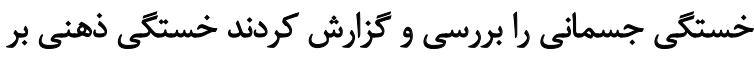

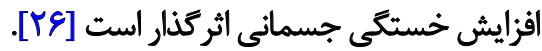

همجنين مشخص شده است كه اعمال اين نوع از تحريكات

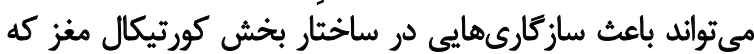

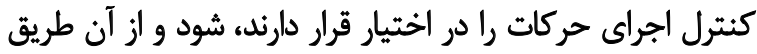

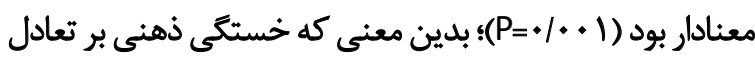

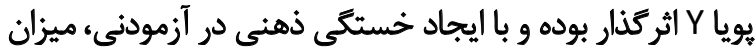

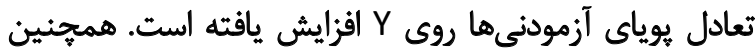

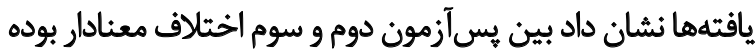

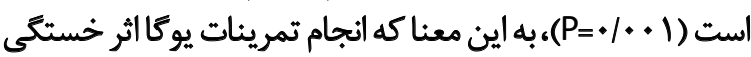

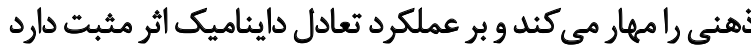

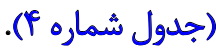

تعادل نيمهيويا روى صفحه يلتفرم

يافتههاى آزمون تحليل واريانس مكرر نشان داد اثر زمان

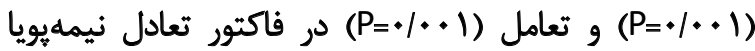

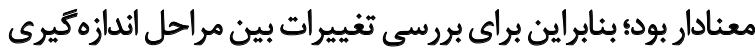

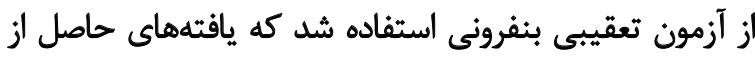

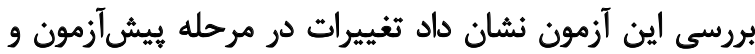

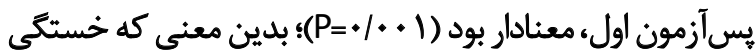

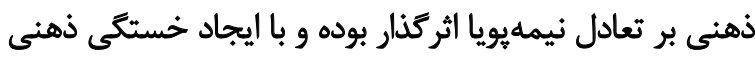

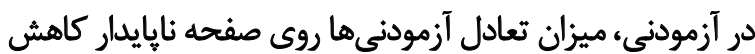

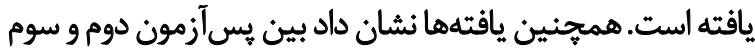

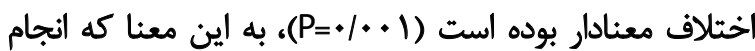

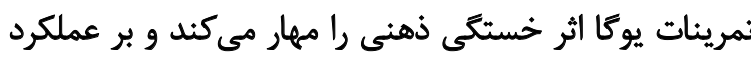
تعادل نيمهيويا اثر مثبت دارد (جدول شماره هات ).

بحث

فراوانى آسيبها بهخصوص در اندام تحتانى در بين ورزشكاران

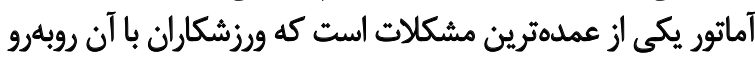

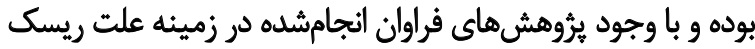

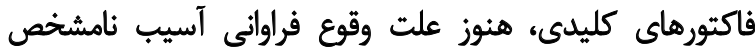

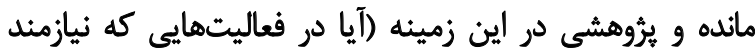

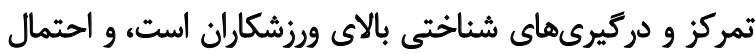

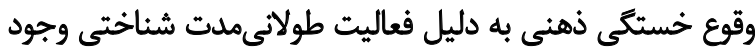

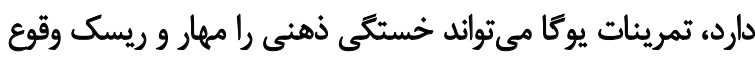

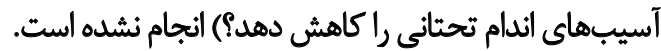

با وجود انجام مطالعات در زمينه اثر تمرينات يوكا بر خستكى مختى

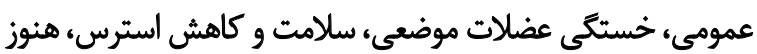

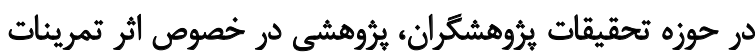

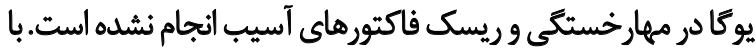
توجه به وجود شكاف تحقيقات، محققين درئ در اين مطالعه بر بر آن شدئند

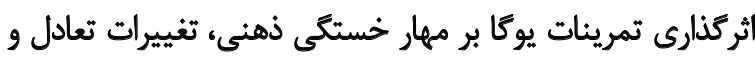
حس عمقى در مردان ورزشكار آماتور را بيازمايند.

نتايج اين يُروهش نشان داد تمرينات يوكا با افزايش توان سازگًارى

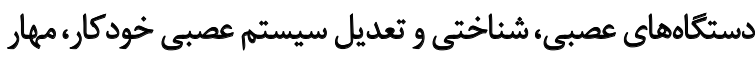

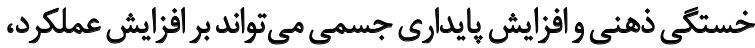
تعادل و حس عمقى مفصل زانو در وضعيت فلكشن و اكستنشن 


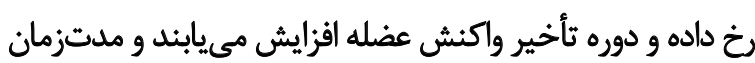

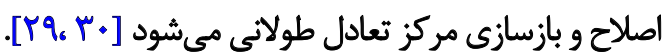

\section{نتيجديّيرى نهايى}

اعمال تمرينات يوكا به طور قابل توجهي باعث بهبود تعادل، حس

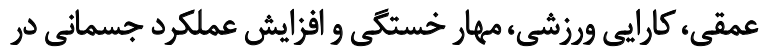

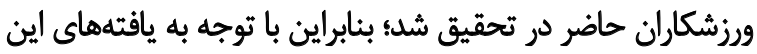

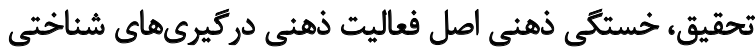

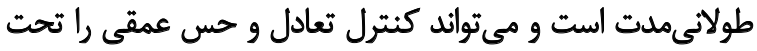

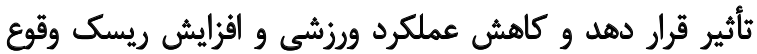

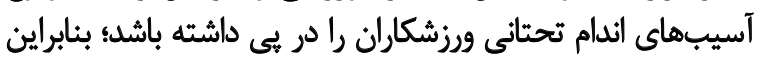

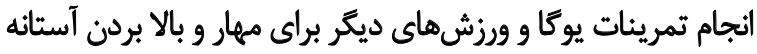

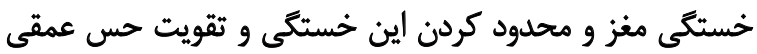

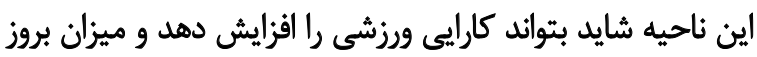

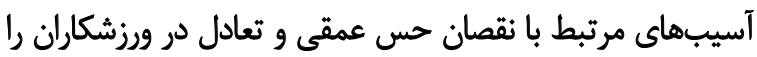

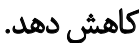

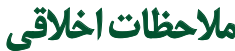

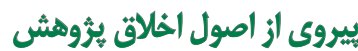

تمام آزمودنىهاى مطالعه، پِّ از تكميل آتاهائه فرمهاى

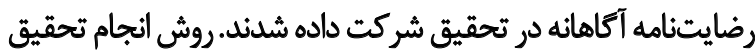

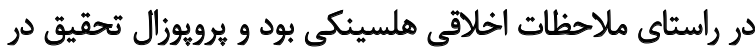

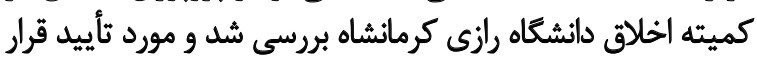

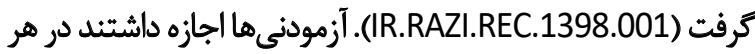
مرحله از تحقيق از ادامه كار سر باز زيند.

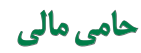

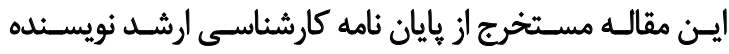

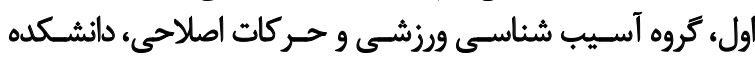

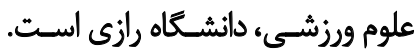

$$
\text { مشاركت نويسندكان }
$$

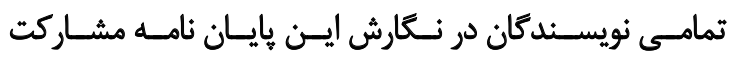

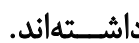

$$
\text { تعارض مناقع }
$$

نويسندكان هيج تعارض منافعى كزارش نكردهاند.

$$
\text { تشكر و قدرداثى ت ت ت }
$$

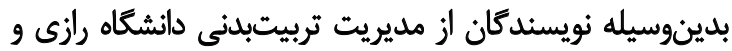

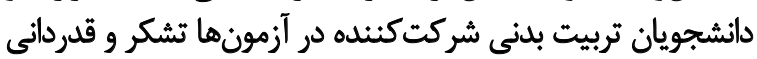

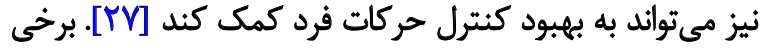

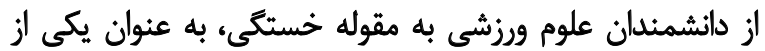

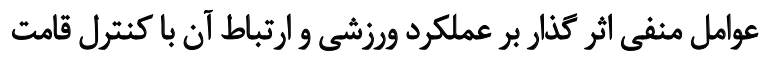

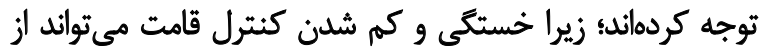

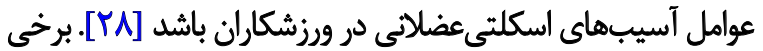

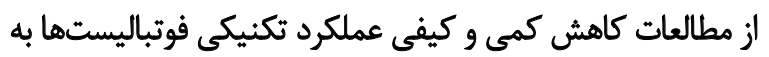

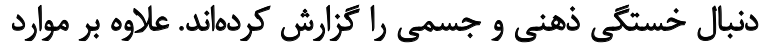

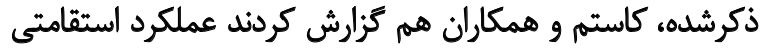

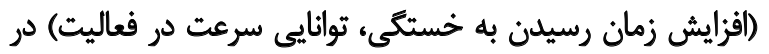

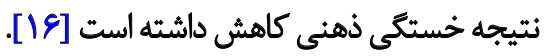

دياتوكاتينكهو و همكاران در تحقيقات خود اثر خستخى

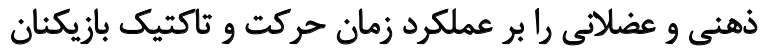

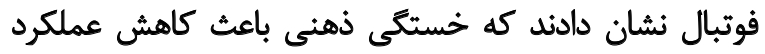

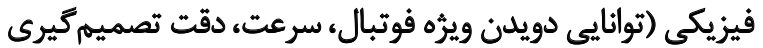

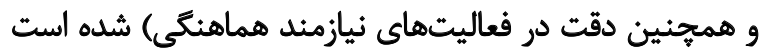

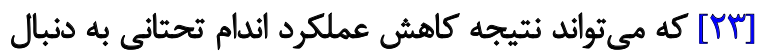
خستكى ذهنى در اين مطالعه را تأييد كند.

علاوه بر آن نتايج اين مطالعه نشان داد تمرينات يوكا باعث

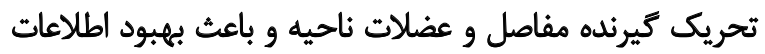

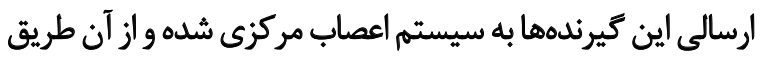

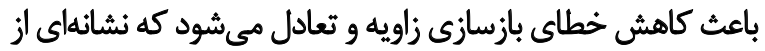

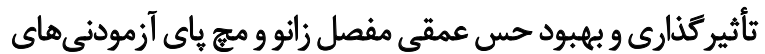

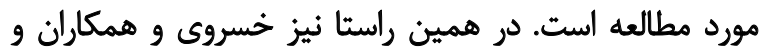

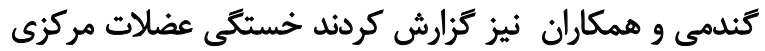

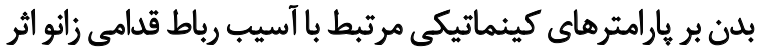

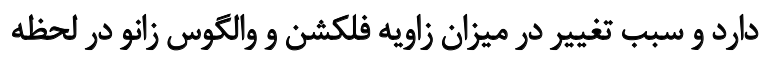

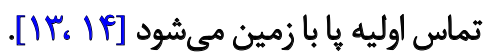

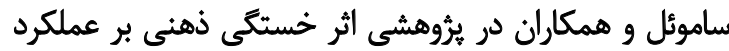

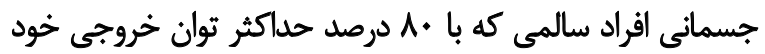

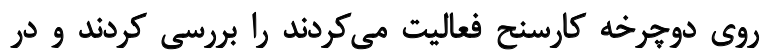

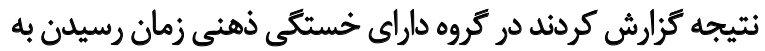

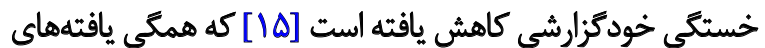
اين مطالعه را تأييد مي كنئد به طور كلى مى توان كفت يوكابه عنوان يك نظريه شخصيت نيز

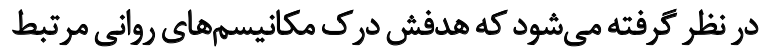

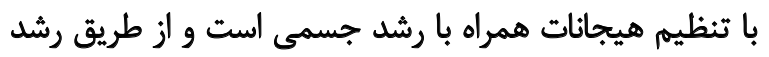

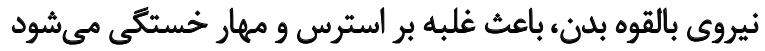

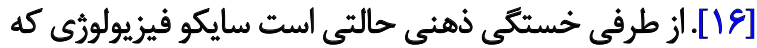

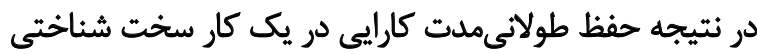

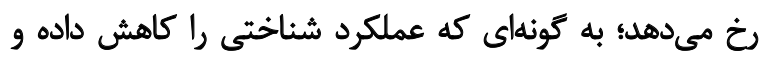

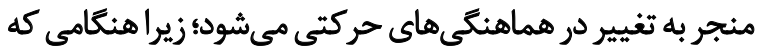

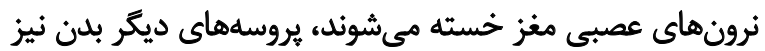

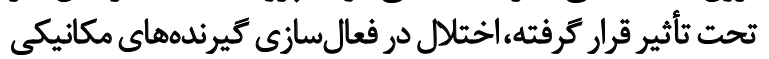




\section{References}

[1] Hashemi J, Chandrashekar N, Jang T, Karpat F, Oseto M, Ekwaro-Osire $S$. An alternative mechanism of non-contact anterior cruciate ligament injury during jump-landing: In-vitro simulation. Exp Mech. 2007; 47(3):347-54. [DOI:10.1007/s11340-007-9043-y]

[2] Shimokochi Y, Shultz SJ. Mechanisms of noncontact anterior cruciate ligament injury. J Athl Train. 2008; 43(4):396-408. [DOI:10.4085/10626050-43.4.396] [PMID] [PMCID]

[3] Letafatkar K, Alizadeh MH, Kordi MR. The effect of exhausting exercise induced fatigue on the double-leg balance of elite male athletes. J Soc Sci. 2009; 5(4):445-51. [DOI:10.3844/jssp.2009.445.451]

[4] Salavaati M. [Study the functional controlling disorders in patients with chronic low back pain and effect of core stabilization training on it (Persian)]. [PhD Thesis]. Tehran: Tarbiat Modares University.

[5] Vaugoyeau M, Viel S, Amblard B, Azulay JP, Assaiante C. Proprioceptive contribution of postural control as assessed from very slow oscillations of the support in healthy humans. Gait posture. 2008; 27(2):294-302. [DOI:10.1016/j.gaitpost.2007.04.003] [PMID]

[6] Ergen E, Ulkar B. Proprioception and Ankle Injuries in Soccer. Clin Sports Med. 2008; 27(1): 195-217. [DOI: 10.1016/j.csm.2007.10.002] [PMID]

[7] Cameron ML, Adams RD, Maher CG. The effect of neoprene shorts on leg proprioception in Australian football players. J Sci Med Sport. 2008; 11(3):345-52. [DOI:10.1016/j.jsams.2007.03.007] [PMID]

[8] Gribble PA, Hertel J, Denegar CR, Buckley WE. The effects of fatigue and chronic ankle instability on dynamic postural control. J Athl Train. 2004; 39(4):321-9. [PMID] [PMCID]

[9] Biedert R. Contribution of the three levels of nervous system motor control: Spinal cord, lower brain, cerebral cortex. In: Lephart SM, Fu FH (Editors), Proprioception and neuromuscular control in joint stability. Champaign: Human Kinetics; 2000. p. 23-31.

[10] Ortiz A, Olson SL, Etnyre B, Trudelle-Jackson EE, Bartlett W, VenegasRios HL. Fatigue effects on knee joint stability during two jump tasks in women. J Strength Cond Res. 2010; 24(4):1019-27. [DOI:10.1519/ JSC.0b013e3181c7c5d4] [PMID] [PMCID]

[11] Smith MR, Zeuwts L, Lenoir M, Hens N, De Jong LMS, Coutts AJ. Mental fatigue impairs soccer-specific decision-making skill. J Sports Sci. 2016; 34(14):1297-304. [DOI:10.1080/02640414.2016.1156241] [PMID]

[12] Marcora SM, Staiano W, Manning V. Mental fatigue impairs physical performance in humans. J Appl Physiol. 2009; 106(3):857-64. [DOI:10.1152/japplphysiol.91324.2008] [PMID]

[13] Gandomi F, Najafi M. Effect of core muscles fatigue on landing mechanic and lower extremity function. J Rehab Med. 2018; 7(4):30-40. [DOI: 10.22037/JRM.2018.110926.1631]

[14] Khosravi S, Eraghi ES, Seydi F. The effect of core muscle functional fatigue on some of kinematics parameters related to $A C L$ injury during single-leg stop-jump task in female athletes. J Rehab Med. 2018; 7(1):1021. [DOI: 10.22037/JRM.2018.110698.1467]

[15] Gonçalves LC, de Souza Vale RG, Barata NJF, Varejão RV, Dantas EHM Flexibility, functional autonomy and Quality of Life (QoL) in elderly yoga practitioners. Arch Gerontol Geriatr. 2011; 53: 158-162. [DOI:10.1016/j. archger.2010.10.028] [PMID]

[16] Raub JA. Psychophysiologic effects of Hatha yoga on musculoskeletal and cardiopulmonary function: A literature review. J Altern Complement Med. 2002; 8(6):797-812. [DOI:10.1089/10755530260511810] [PMID]
[17] Villien $F, Y u$ M, Barthelemy $P$, Jammes $Y$. Training to yoga respiration selectively increases respiratory sensation in healthy man. Respir Phys iol Neurobiol. 2005; 146(1):85-96. [DOI:10.1016/j.resp.2004.11.010] [PMID]

[18] Coughlan GF, Fullam K, Delahunt E, Gissane C, Caulfield BM. A comparison between performance on selected directions of the star excursion balance test and the $Y$ balance test. J Athl Train. 2012; 47(4):366-71. [DOI:10.4085/1062-6050-47.4.03] [PMID] [PMCID]

[19] Zech A, Meining S, Hötting K, Liebl D, Mattes K, Hollander K. Effects of barefoot and footwear conditions on learning of a dynamic balance task: A randomized controlled study. Eur J Appl Physiol. 2018; 118(12):2699706. [DOI:10.1007/s00421-018-3997-6] [PMID]

[20] Herrington L. Knee-joint position sense: The relationship between open and closed kinetic chain Tests. J sport rehabil. 2005; 14(4):356-62. [DOI:10.1123/jsr.14.4.356]

[21] Clapis PA, Davis SM, Davis RO. Reliability of inclinometer and goniometric measurements of hip extension flexibility using the modified Thomas test. Physiother Theory Pract. 2008; 24(2):135-41. [DOI:10.1080/09593980701378256] [PMID]

[22] Fouladi R, Nasseri N, Rajabi R, Geranmayeh M. [Joint position sense of the knee in healthy female athletes across the menstrual cycle (Persian)]. Koomesh. 2010; 12(1):31-8. http://koomeshjournal.semums ac.ir/article-1-957-en.htm

[23] Coutinho D, Gonçalves B, Wong DP, Travassos B, Coutts AJ, Sampaio $J$. Exploring the effects of mental and muscular fatigue in soccer players' performance. Hum Mov Sci. 2018; 58:287-96. [DOI:10.1016/j.humov.2018.03.004] [PMID]

[24] Martin K, Meeusen R, Thompson KG, Keegan R, Rattray B. Mental fatigue impairs endurance performance: $A$ physiological explanation. Sports Med. 2018; 48(9):2041-51. [DOI:10.1007/s40279-018-0946-9] [PMID]

[25] Baroni BM, Wiest MJ, Generosi RA, Vaz MA, Leal Junior ECP. Effect of muscle fatigue on posture control in soccer Players during the short-pass movement. Rev bras cineantropom desempenho hum. 2011; 13(5):34853. https://doi.org/10.5007/1980-0037.2011v13n5p348.

[26] Mehta RK, Parasuraman R. Effects of mental fatigue on the development of physical fatigue: A neuroergonomic approach. Hum factors. 2014; 56(4):645-56. [DOI:10.1177/0018720813507279] [PMID]

[27] Carel C, Loubinoux I, Boulanouar K, Manelfe C, Rascol O, Celsis P, et al. Neural substrate for the effects of passive training on sensorimotor cortical representation: A study with functional magnetic resonance imaging in healthy subjects. J Cereb Blood Flow Metab. 2000; 20(3):478-84. [DOI:10.1097/00004647-200003000-00006] [PMID]

[28] Guskiewicz KM, Perrin DH. Research and clinical applications of assessing balance. J Sport Rehabil. 1996; 5(1):45-63. [DOI:10.1123/jsr.5.1.45]

[29] Cortes N, Quammen D, Lucci S, Greska E, Onate J. A functional agility short-term fatigue protocol changes lower extremity mechanics. J Sports Sci. 2012; 30(8):797-805. [DOI:10.1080/02640414.2012.671528 ] [PMID] [PMCID]

[30] Earl JE, Hertel J. Lower-extremity muscle activation during the Star Excursion Balance tests. J Sport Rehabil. 2001; 10(2):93-104. [DOI:10.1123/ jsr.10.2.93] 
This Page Intentionally Left Blank 